\title{
Error Compensation on Probe Parameters of Articulated Arm CMM
}

\author{
Dateng Zheng, Gang Zhao , Zhongyue Xiao, Zhiyang Luo, Taiping Zhou and Jing Zhang \\ Jinggangshan University College of Mechanical \& Electrical Engineering, Ji'an, 343009, China
}

\begin{abstract}
The error compensations on probe parameters were proposed to improve the measurement accuracy of an articulated arm coordinate measuring machines (AACMM). An AACMM's probe parameters were analyzed and a measurement model including probe parameters solely was built. A simple cone socket components were used as a calibration tool and the LM algorithm was edited to solve the probe parameters. The results show that the average measurement error dealt with the probe parameters recalibration is $0.044 \mathrm{~mm}$, and its standard deviation is $0.050 \mathrm{~mm}$. They have increased by about $31 \%$ and $33 \%$ than those without recalibration.
\end{abstract}

\section{Introduction}

An articulated arm coordinate measuring machine (AACMM) [1] is one kind of CMMs. Comparing with conventional orthogonal CMM, its measurement precision is lower for its complicated structure in series and others. How to improve AACMM's measurement accuracy is an objective for researchers. Wang Pingping built a measurement model with 25 parameters [2]. Wang Bin built a measurement equation with 37 errors and simplified the model with structuring recombination spherical coordinate [3]. Shang Ping analyzed the error source roundly, built the measurement equation with 60 errors [4]. Zhu Lianqing built a measurement model with 27 parameters for partly-bonded articulated arm coordinate measuring machine [5]. $\mathrm{Hu} \mathrm{Yi}$ and Jorge Santolaria studied the thermal deformation error, and they built the error models including thermal deformation error using different method, respectively [6-7]. Huang Kui established a kinematic model involving 6 degrees of freedom (DOF) parameters with local product of exponentials formula[8]. Wang Wen introduced a simulated annealing algorithm to identify the eccentricity parameter s of the AACMM [9]. In our previous study, we put forward a spatial error model based on functional networks and validated the new error model and the approximate algorithm on the results of precision measurement experiment [10], and adopted the SVM method to find a way to build the different error model[11]. The existing researches help to improve AACMM's measurement accuracy, but they don't consider the factors of probe parameters separately.

As a kind of portable coordinate measurement equipment, an AACMM's probe needs to be disassembled when it is carried to another job site. The probe sometimes needs to be replaced during use. The probe parameters from measurement model are changed when it is installed in these cases. So we must find an advantageous way to recalibrate these parameters. In this study, we discuss how to build the probe parameter model, to do sample experiments simply and to calibrate the probe parameters. It is significant and helpful for primary operators because the sample method for the probe parameters is simpler than those for the whole structural parameters, and particularly the former's calibration time is shorter.

\section{Probe parameter model}

AACMM analogs the human's articulated structure which is mainly mechanically composed of a base, an upright column, two measuring arms, six rotary joints with circular grating encoders and a probe, as shown in Fig. 1.

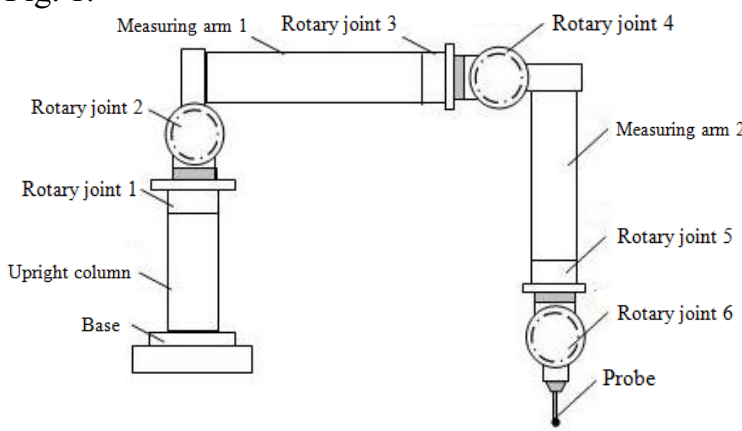

Figure 1. Structural diagram of AACMM

AACMM's measurement model is often built by the D-H (Denavit and Hartenberg) method [12], as shown in equation (1) 


$$
\begin{aligned}
& {\left[\begin{array}{l}
x_{z} \\
y_{z} \\
z_{z} \\
1
\end{array}\right]=\prod_{i=1}^{6}\left[\begin{array}{cc}
\cos \left(\theta_{i}+\theta_{i 0}\right) & -\sin \left(\theta_{i}+\theta_{i 0}\right) \cos \alpha_{i} \\
\sin \left(\theta_{i}+\theta_{i 0}\right) & \cos \left(\theta_{i}+\theta_{i 0}\right) \cos \alpha_{i} \\
0 & \sin \alpha_{i} \\
0 & 0
\end{array}\right.} \\
& \left.\begin{array}{cc}
\sin \left(\theta_{i}+\theta_{i 0}\right) \sin \alpha_{i} & I_{i} \cos \left(\theta_{i}+\theta_{i 0}\right) \\
-\cos \left(\theta_{i}+\theta_{i 0}\right) \sin \alpha_{i} I_{i} \sin \left(\theta_{i}+\theta_{i 0}\right) \\
\cos \alpha_{i} & d_{i} \\
0 & 1
\end{array}\right]\left[\begin{array}{l}
0 \\
0 \\
z_{z} \\
1
\end{array}\right]
\end{aligned}
$$

Where $\theta_{\mathrm{i}}(\mathrm{i}=1,2, \ldots, 6)$ are joint variables, $\theta_{\mathrm{i} 0}(\mathrm{i}=1,2, \ldots, 6)$ are joint zero errors; $\alpha_{\mathrm{i}}(\mathrm{i}=1,2, \ldots, 6)$ are torsional angles from joint axis to joint axis, including their perpendicularity errors; $l_{\mathrm{i}}(\mathrm{i}=1,2, \ldots, 6)$ are lengths of rod pieces, including their errors; and $d_{\mathrm{i}}(\mathrm{i}=1,2, \ldots, 6)$ are offsets of rod pieces, including their errors.

In this study, the AACMM's structural parameters are shown in table 1("-"denotes that the parameter cannot be calibrated).

\begin{tabular}{|c|c|c|c|c|}
\hline Parameter & $\theta_{i 0}(\mathrm{rad})$ & $\alpha_{i}(\mathrm{rad})$ & $l_{i}(\mathrm{~mm})$ & $d_{i}(\mathrm{~mm})$ \\
\hline Joint 1 & - & $\begin{array}{c}- \\
1.5704\end{array}$ & 29.3705 & - \\
\hline Joint 2 & $\begin{array}{c}- \\
2.7770\end{array}$ & 1.5731 & -30.1459 & -0.3681 \\
\hline Joint 3 & $\begin{array}{c}- \\
4.3312\end{array}$ & $\begin{array}{c}- \\
1.5711\end{array}$ & 27.1532 & 300.5980 \\
\hline Joint 4 & $\begin{array}{c}- \\
4.5854\end{array}$ & 1.5704 & -27.0528 & -0.4637 \\
\hline Joint 5 & $\begin{array}{c}- \\
2.7668\end{array}$ & $\begin{array}{c}- \\
1.5705\end{array}$ & 26.6343 & 278.6602 \\
\hline Joint 6 & $\begin{array}{c}- \\
0.9410\end{array}$ & - & 170.8003 & -0.8118 \\
\hline
\end{tabular}

Table 1. Calibration values of structural parameters

When a probe is replaced and reinstalled, the probe parameters are changed subsequently. Apparently, the probe parameters which need to be calibrated are $\theta_{60}, l_{6}$ and $d_{6}$. The Figure 2 made clear that the parameters of $\theta_{60}, l_{6}$ and $d_{6}$ are before replace. The parameters of $\theta_{60}{ }^{\prime}$, $l_{6}{ }^{\prime}$ and $d_{6}{ }^{\prime}$ are after replace.

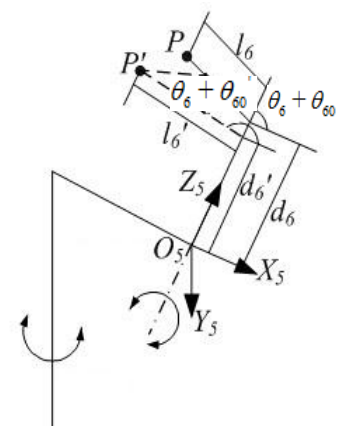

Figure 2. Probe parameters change sketch
The other parameters except the probe parameters in equation (1) are not changed only if the probe is replaced and reinstalled. So the probe parameter model is shown in equation (2).

$$
\begin{aligned}
& {\left[\begin{array}{l}
x_{z} \\
y_{z} \\
z_{z} \\
1
\end{array}\right]=\prod_{i=1}^{5}\left[\begin{array}{cc}
\cos \left(\theta_{i}+\theta_{i 0}\right) & -\sin \left(\theta_{i}+\theta_{i 0}\right) \cos \alpha_{i} \\
\sin \left(\theta_{i}+\theta_{i 0}\right) & \cos \left(\theta_{i}+\theta_{i 0}\right) \cos \alpha_{i} \\
0 & \sin \alpha_{i} \\
0 & 0
\end{array}\right.} \\
& \left.\begin{array}{cc}
\sin \left(\theta_{i}+\theta_{i 0}\right) \sin \alpha_{i} & I_{i} \cos \left(\theta_{i}+\theta_{i 0}\right) \\
-\cos \left(\theta_{i}+\theta_{i 0}\right) \sin \alpha_{i} I_{i} \sin \left(\theta_{i}+\theta_{i 0}\right) \\
\cos \alpha_{i} & d_{i} \\
0 & 1
\end{array}\right]
\end{aligned}
$$$$
\times\left[\begin{array}{cc}
\cos \left(\theta_{6}+\theta_{60}\right) & -\sin \left(\theta_{6}+\theta_{60}\right) \cos \alpha_{6} \\
\sin \left(\theta_{6}+\theta_{60}^{\prime}\right) & \cos \left(\theta_{6}+\theta_{60}\right) \cos \alpha_{6} \\
0 & \sin \alpha_{6} \\
0 & 0
\end{array}\right.
$$

$$
\left.\begin{array}{cc}
\sin \left(\theta_{6}+\theta_{60}\right) \sin \alpha_{6} & I_{6}^{\prime} \cos \left(\theta_{6}+\theta_{60}^{\prime}\right) \\
-\cos \left(\theta_{6}+\theta_{60}\right) \sin \alpha_{6} I_{6}^{\prime} & \sin \left(\theta_{6}+\theta_{60}\right) \\
\cos \alpha_{6} & \sigma_{6}^{\prime} \\
0 & 1
\end{array}\right] \times\left[\begin{array}{l}
0 \\
0 \\
z_{7} \\
1
\end{array}\right]
$$

\section{Calibration experiment}

In order to make sample manipulate simply, we use the cone socket components as a calibration tool in this work, as shown in Fig. 3. They are mainly composed of a disc and a metallic rod. The disc has five threaded holes which are fixed the rods. One end of the rod has a standard tapered bore.

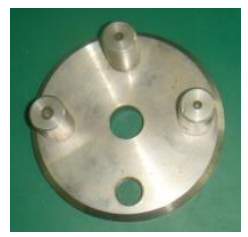

Figure 3. Cone socket components

Before sampling, we disassembled the AACMM's probe, and then assembled the same probe. We fixed the cone socket to a certain location. And then we used the AACMM's probe to detect the standard tapered bore as many postures as possible from various directions successively. Xi Si drew a conclusion that the max measurement error and standard deviation would tend to be stable when the samples reached 200 times in his master's thesis [13]. So we collected 200 sets of data to 
calibrate the probe parameters $\left(\theta_{60}{ }^{\prime}, l_{6}{ }^{\prime}, d_{6}{ }^{\prime}\right)$. The calibration experiment is shown in Fig. 4.

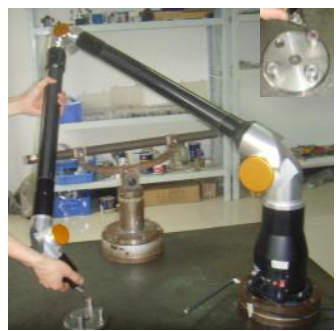

Figure 4. Data collection in calibration experiment

\section{Parameters calibration}

The three probe parameters $\left(\theta_{60}{ }^{\prime}, l_{6}{ }^{\prime}, d_{6}{ }^{\prime}\right)$ need to be calibrated to obtain precise values. We had to construct a calibration algorithm to solve the probe parameters after we collected 200 sets of samples. Many calibration algorithms had been widely studied by people all the time. CHEN Zhenyu presented a genetics-based calibration algorithm for the structural parameters of multi-joints 3D scanner [14]. WANG Xueying further presented an improved genetic algorithm for the parallel recombination simulated annealing [15]. Wang Pingping adopted the least-square parameters identification method to calibrate the AACMM's structure parameters with the standard provided by a high-accuracy orthogonal CMM [2]. GAO Guanbin presented a modified simulated annealing algorithm to identify the structural parameters of the AACMMs [16]. In terms of an algorithm's speed, effectiveness and stability, a comparative analysis was made to show that Levenberg-Marquardt(LM) algorithm as one of nonlinear least square method was the least time-consuming and produced the most stable outputs[17]. So we chose the LM calibration algorithm in this work.

One set of sample is the reading value $\left(\theta_{1}, \theta_{2}, \theta_{3}, \theta_{4}\right.$, $\left.\theta_{5}, \theta_{6}\right)$ from circular grating encoders. We can calculate the coordinate value $\mathrm{P}(\mathrm{X}, \mathrm{Y}, \mathrm{Z})$ if the three probe parameters are calibrated. The cone socket is fixed in one location when we used the AACMM's probe to detect the standard tapered bore. So 200 coordinate values are theoretically same. The equation (3) can be built as follows.

$$
\left\|P_{i} P_{j}\right\|=0(i \neq j)
$$

It is easy to know that the equation (3) is not equal if the three probe parameters are not yet calibrated. But the left expression is equal to a minimal value, so the optimal object can be expressed as follows.

$$
\min _{\theta_{60}, l_{6}, d_{6}^{\prime} \in R} S(\boldsymbol{b})=\sum_{i=1}^{n} \sum_{j=i}^{n}\left\|P_{i} P_{j}\right\|^{2}=\|\boldsymbol{F}(\boldsymbol{b})\|_{2}^{2}
$$

The LM calibration algorithm was applied in this work, the detailed solving steps were as follows.

(1) Choose an initial damping factor $\mu=\mu 00$ and growth factor $\tau>1$.

(2) Give an initial calibration parameter vector $k^{0}$, specify the accuracy $\varepsilon>0$, and set calculation step flag $\mathrm{t}=0$.
(3) Calculate the calibration function $f^{\mathrm{t}}=f^{(\mathrm{kt})}$, residual function $V^{\mathrm{t}}=V^{(\mathrm{kt})}$, and $S^{\mathrm{t}}=S^{(\mathrm{kt})}=\left(f^{\mathrm{t}}\right) \mathrm{T} \quad\left(V^{\mathrm{t}}\right)$, function $\left(f^{\mathrm{t}}\right)^{\mathrm{T}}$ is a transition matrix of $f^{\mathrm{t}}$.

(4) Calculate Jacobian matrix $\boldsymbol{A}^{t}$

$$
A^{t}=\left[\frac{\partial f_{u}\left(k^{t}\right)}{\partial k^{t}}\right] \text {, }
$$

calculate $\left(\boldsymbol{A}^{t}\right)^{\mathrm{T}}\left(\boldsymbol{A}^{t}\right)$.

(5) Calculate

(6) Solve

$$
g^{t}=\nabla S\left(k^{t}\right)=\left(A^{t}\right)^{T} V^{t}
$$

equation to get pt:

$$
\left(\left(A^{t}\right)^{T} A^{t}+\mu_{0}^{t} I\right) p^{t}=-g^{t} .
$$

(7) Calculate

$k^{\mathrm{t}+1}=k^{\mathrm{t}}+p^{\mathrm{t}}$.

(8) Calculate

$\mathrm{V}^{\mathrm{t}+1}=\mathrm{V}\left(\mathrm{k}^{\mathrm{t}+1}\right), \mathrm{S}^{\mathrm{t}+1}=\mathrm{S}\left(\mathrm{k}^{\mathrm{t}+1}\right)$.

(9) If $S^{\mathrm{t}+1} \leq S^{\mathrm{t}}$, then $u_{0}{ }^{\mathrm{t}}=u_{0}{ }^{\mathrm{t}} / \tau$, go to (6).

Test: if $S^{\mathrm{t}+1} \leq S^{\mathrm{t}}$, then $u_{0}{ }^{\mathrm{t}}=u_{0}{ }^{\mathrm{t}} / \tau$;

if $S^{\mathrm{t}+1} \leq S^{\mathrm{t}}$, then $u_{0}{ }^{\mathrm{t}}=u_{0}{ }^{\mathrm{t}}$.

(10) If $S^{t+1}>S^{t}$, then $u_{0 n}{ }^{t}=\tau^{n} u_{0}{ }^{t}$.

Test: set $\mathrm{n}=1,2, \ldots$, in turn, and calculate $u_{0 n}{ }^{t}$ respectively, go to (6).

If $S_{0 n}{ }^{t+1} \leq S^{t}$, then $u_{0}{ }^{t}=u_{0 n}{ }^{t}$.

(11) Check termination criterion, if satisfy the criterion, then output $\mathrm{k}^{*}=\mathrm{kt}+1$, and stop calculation; if not satisfy the criterion, then $\mathrm{t}=\mathrm{t}+1, k^{\mathrm{t}}=k^{\mathrm{t}+1}, S^{\mathrm{t}}=S^{\mathrm{t}+1}$, go to (3).

In this work, $\mathrm{u}_{0}^{0}=0.01, \tau=3, \varepsilon=0.01, \mathrm{~m}=200$.

We edited the algorithm in MATLAB software to solve the probe parameters. The calibration results are shown in Tables 2 after 24 iterations.

Table 2. Probe parameter values before and after calibration

\begin{tabular}{ccc}
\hline State & Probe parameter & value \\
\hline \multirow{3}{*}{ Before calibration } & $\theta_{60}$ & -0.9410 \\
& $l_{6}$ & 170.8003 \\
& $d_{6}$ & -0.8118 \\
\hline \multirow{3}{*}{ After calibration } & $\theta_{60}{ }^{\prime}$ & -0.9401 \\
& $l_{6}{ }^{\prime}$ & 170.5342 \\
& $d_{6}{ }^{\prime}$ & -0.9231 \\
\hline
\end{tabular}

\section{Precision comparison}

In order to verify calibration effect, we used an AACMM to measure a standard rod whose length is $100.2500 \mathrm{~mm}$. We collected 100 sets of data $\left(\theta_{1}, \theta_{2}, \theta_{3}, \theta_{4}, \theta_{5}, \theta_{6}\right)$. By substituting the parameters of Table 1 into equations (1) and the probe parameters $\left(\theta_{60}{ }^{\prime}, l_{6}{ }^{\prime}, d_{6}{ }^{\prime}\right)$ of Table 2 after calibration into equations (2), we can simply compare the effect. The results are shown in Figure 5. 


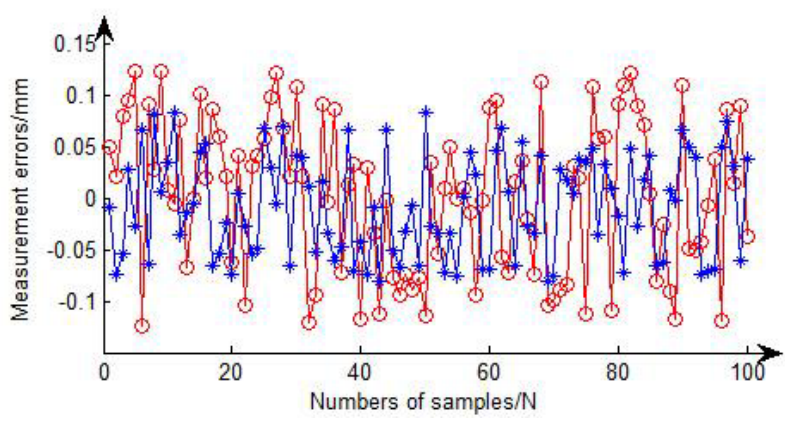

Figure 5. Measurement errors comparison

The average measurement error before the probe parameters are calibrated (red circles) is $0.064 \mathrm{~mm}$, and its standard deviation is $0.075 \mathrm{~mm}$. The average measurement error after the probe parameters are calibrated (blue stars) is $0.044 \mathrm{~mm}$, and its standard deviation is $0.050 \mathrm{~mm}$. It is clear that the measurement accuracy of AACMM dealt with the probe parameters recalibration is superior to the accuracy of no calibration.

\section{Conclusions}

We analyzed an AACMM's probe parameters and built its measurement model. We chose a simple cone socket components as a calibration tool and did a convenient calibration experiment. We edited the LM algorithm in MATLAB software to solve the probe parameters. The results show that the average measurement error dealt with the probe parameters recalibration is $0.044 \mathrm{~mm}$, and its standard deviation is $0.050 \mathrm{~mm}$.

\section{Acknowledgements}

We gratefully acknowledge the project 51265017 supported by National Natural Science Foundation of
China and the project 20131828 by Ji'an Municipal Natural Science Foundation, which are the basis for the proposed achievements.

\section{References}

1. G.X. ZHANG. Aviation Precision Manufacturing Technology, 44,3( 2008).

2. P.P.Wang, Y.T.Fei, S.W. Lin. Journal of Xi'An Jiaotong University,40,3(2006).

3. B. WANG, S.G. Liu, X.Y. WANG. Measurement Technique, 5,(2007).

4. P. Shang, Y.T.Fei. Tool Engineering, 8,(2009).

5. L.Q. Zhu, W.X. Li, Z.K. Pan, et al. Chinese Journal of Scientific Instrument,35,3 (2014).

6. Y. Hu, Y.T.Fei, W.T. Cheng. JOURNAL OF MECHANICAL ENGINEERING,47,24(2011).

7. J. Santolaria, J.A.Yagüe, R.Jiménez, J.J.Aguilar. Precision Engineering,33,4(2009).

8. K. HUANG , J.H. Mo, L.H. Yu, et al. Journal of Xi'an Jiaotong University, 44,8(2010).

9. W. WANG, K. Lin, G.B. Gao,Z.C. Chen. Optics and Precision Engineering, 18,1(2010).

10. D.T. Zheng, Y.T.Fei. Journal of Mechnical Engineering, 46,10(2010).

11. D.T. Zheng, Y.G. Hu. Measurement, 45,3(2012).

12. L.D. Yu, W.T. Cheng, Y.T.Fei. Journal of University of Science and Technology of China, 39,(2009).

13. S. Xi. Hefei University of technology,2013.

14. Z.Y. Chen, C.S. Fu, D.H. Li. Infrared and Laser Engineering, 32,5(2003).

15. X.Y. Wang, S.G. Liu, H.T. Zhang, et al. Key Engineering Materials, 161,(2008).

16. G.B. Gao, W. WANG, K. Lin, Z.C. Chen. Optics and Precision Engineering, 15,8(2009).

17. W.T. Cheng, L.D. Yu, Y.T.Fei. Journal of University of Science and Technology of China, 41,1(2011). 